LAPP-EXP-2004-14

October 2004

\title{
Reconstruction of fundamental SUSY parameters at LHC and at a future linear collider
}

\author{
R. Lafaye \\ CERN, Geneva, Switzerland, on leave from LAPP, Annecy, France \\ T. Plehn \\ CERN Theory Division, Geneva, Switzerland \\ D. Zerwas \\ LAL, Orsay, France
}

Presented at the 32nd International Conference on High Energy Physics ICHEP 2004, Beijing (China) August 16-22, 2004 
Beijing ICHEP 2004

Conference paper

\title{
Reconstruction of fundamental SUSY parameters at LHC and at a future Linear Collider
}

\author{
REMI LAFAYE ${ }^{1}$, TILMAN PLEHN ${ }^{2}$, AND DiRK ZERWAS ${ }^{3}$ \\ ${ }^{1}$ CERN, Geneva, Switzerland, on leave from LAPP, Annecy, France \\ ${ }^{2}$ CERN Theory Division, Geneva, Switzerland \\ ${ }^{3}$ LAL, Orsay, France
}

\begin{abstract}
Unveiling the mechanism leading to the breaking of supersymmetry is among the outstanding questions for future colliders. To achieve this goal, models will need to be scrutinized and their parameters assessed. Global fitting tools, like Fittino and SFitter, have been developed and set a robust framework for such analyses.

Using the SPS1a snowmass point as an example for the SUSY and Higgs particles that could be observed at the LHC and at a future TeV Linear Collider, we have studied the determination and its precision of MSUGRA parameters from the measurements expected in this point. While the LHC will provide the first measurement of the parameters, the Linear Collider will increase their precision by an order of magnitude.

However, when moving to the unconstrained weak-scale MSSM, measurements from the LHC, such as the gluino and squarks masses and couplings, and from the LC, such as charginos and high precision slepton mass measurements, are necessary to reconstruct the Lagrangian with the best available precision.

Using a set of hypothetical measurements at LHC and at a future LC, we will show how these colliders probe different sectors of the MSSM Lagrangian and how this complementarity increases our handle on the determination of the weak-scale parameters of the Lagrangian. In fact, the combination of the measurements of the LHC and the LC is essential to probe the complete MSSM weak-scale lagrangian.
\end{abstract}

Note: although this conference paper may describe the work upon which the corresponding talk and proceedings will be based, it might not completly reflect their actual contents.

\section{Introduction}

If supersymmetry [1] or any other high-scale extension of the Standard Model is discovered, it will be crucial to determine its fundamental high-scale parameters from weakscale measurements [2]. The LHC and future Linear Colliders will provide us with a wealth of measurements $[3,4]$, which due to their complexity require proper treatment to unravel the corresponding high-scale physics. Even in the general weak-scale minimal supersymmetric extension of the standard model (MSSM [5]) without any unification or SUSY breaking assumptions some of the measurements of masses and couplings 


\begin{tabular}{|l|c|cc|cc|cc|}
\hline & $m_{\text {SPS1a }}$ & $\Delta_{L H C}^{e x p}$ & $\Delta_{L H C}^{e x p+t h}$ & $\Delta_{L C}^{e x p}$ & $\Delta_{L C}^{e x p+t h}$ & $\Delta_{L H C+L C}^{e x p}$ & $\Delta_{L H C+L C}^{\text {exp }+t h}$ \\
\hline \hline$h$ & 111.6 & 0.25 & 3.01 & 0.05 & 3.0 & 0.05 & 3.0 \\
$H$ & 399.6 & & & 1.5 & 3.35 & 1.5 & 3.35 \\
$A$ & 399.1 & & & 1.5 & 3.35 & 1.5 & 3.35 \\
$H+$ & 407.1 & & & 1.5 & 3.35 & 1.5 & 3.35 \\
\hline
\end{tabular}

Table 1: Errors for the mass determination in SPS1a, taken from $[6,7,19]$. Shown are the nominal parameter values and the experimental and sum of experimental and theoretical uncertainties for the LHC alone, the LC alone, and a combined LHC+LC analysis. All values are given in $\mathrm{GeV}$.

are not independent measurements; moreover, linking supersymmetric particle masses to weak-scale SUSY parameters involves non-trivial mixing to mass eigenstates in essentially every sector of the theory. On top of that, for example in gravity mediated SUSY breaking scenarios (mSUGRA/cMSSM) a given weak-scale SUSY parameter will always be sensitive to several high-scale parameters which contribute through renormalization group running. Therefore, a fit of the model parameters using all experimental information available will lead to the best sensitivity and make the most efficient use of the information available.

In a fit, the allowed parameter space might not be sampled completely. To avoid boundaries imposed by non-physical parameter points, which can confine the fit to a 'wrong' parameter region, combining the fit with an initial evaluation of a multi-dimensional grid is the optimal approach.

In the general MSSM the weak-scale parameters can vastly outnumber the collider measurements, so that a complete parameter fit is not possible and one has to limit oneself to a consistent subset of parameters. In SFITTER both grid and fit are realised and can be combined. This way, one can ultimately eliminate all dependence on the starting point of the parameter determination. SFITTER also includes a general correlation matrix and the option to exclude parameters of the model from the fit/grid by fixing them to a value. Additionally, SFITTER includes the option to apply a Gaussian smearing to all observables before they enter the fit/grid in order to simulate realistically experimental measurements. In this preliminary study, however, correlations and systematic uncertainties are neglected and the central values are used for the measurements.

Currently, SFITTER uses the predictions for the supersymmetric masses provided by SUSPECT [8], but the conventions of the SUSY Les Houches accord [9] allow us to interface other programs. The branching ratios and $e^{+} e^{-}$production cross sections are provided by MSMlib [10], which has been used extensively at LEP and cross checked with Ref. [11]. The next-to-leading order hadron collider cross sections are computed using PROSPINO [12]. The fitting program uses the MINUIT package [13]. The determination of $\chi^{2}$ includes a general correlation matrix between measurements. In its next version SFITTER will be interfaced with the improved branching fraction determination of SDECAY [14], as well as alternative renormalization group codes like SoftSUSY [16], ISAJET [17] or SPHENO [18]. 


\begin{tabular}{|l|c|cc|cc|cc|}
\hline & $m_{\text {SPS1a }}$ & $\Delta_{L H C}^{e x p}$ & $\Delta_{L H C}^{\text {exp }+ \text { th }}$ & $\Delta_{L C}^{\text {exp }}$ & $\Delta_{L C}^{\text {exp }+ \text { th }}$ & $\Delta_{L H C+L C}^{\text {exp }}$ & $\Delta_{L H C+L C}^{\text {exp+th }}$ \\
\hline \hline$\chi_{1}^{0}$ & 97.03 & 4.8 & 4.81 & 0.05 & 0.30 & 0.05 & 0.30 \\
$\chi_{2}^{0}$ & 182.9 & 4.7 & 4.93 & 1.2 & 1.92 & 0.08 & 1.50 \\
$\chi_{3}^{0}$ & 349.2 & & & 4.0 & 5.0 & 4.0 & 5.0 \\
$\chi_{4}^{0}$ & 370.3 & 5.1 & 5.92 & 4.0 & 5.0 & 2.3 & 3.78 \\
$\chi_{1}^{ \pm}$ & 182.3 & & & 0.55 & 1.32 & 0.55 & 1.32 \\
$\chi_{2}^{ \pm}$ & 370.6 & & & 3.0 & 3.91 & 3.0 & 3.91 \\
\hline$\tilde{g}$ & 615.7 & 8.0 & 15.26 & & & 6.4 & 14.53 \\
\hline$\tilde{t}_{1}$ & 411.8 & & & 2.0 & 13.15 & 2.0 & 13.15 \\
$\tilde{b}_{1}$ & 520.8 & 7.5 & 18.03 & & & 5.7 & 17.36 \\
$\tilde{b}_{2}$ & 550.4 & 7.9 & 18.20 & & & 6.2 & 17.53 \\
\hline$\tilde{q}_{1}$ & 551.0 & 19.0 & 24.21 & & & 16.0 & 21.93 \\
$\tilde{q}_{2}$ & 570.8 & 17.4 & 26.51 & & & 9.8 & 22.27 \\
\hline$\tilde{\tau}_{1}$ & 135.5 & 6.5 & 6.55 & 0.3 & 0.85 & 0.3 & 0.85 \\
$\tilde{\tau}_{2}$ & 207.9 & & & 1.1 & 1.49 & 1.1 & 1.49 \\
$\tilde{\mu}_{1}$ & 144.9 & 4.8 & 4.84 & 0.2 & 0.63 & 0.2 & 0.63 \\
$\tilde{\mu}_{2}$ & 204.2 & 5.0 & 4.92 & 0.5 & 1.21 & 0.5 & 1.21 \\
$\tilde{e}_{1}$ & 144.9 & 4.8 & 5.04 & 0.05 & 0.60 & 0.05 & 0.60 \\
$\tilde{e}_{2}$ & 204.2 & 5.0 & 5.12 & 0.2 & 1.12 & 0.2 & 1.12 \\
$\tilde{\nu}_{e}$ & 188.2 & & & 1.2 & 1.63 & 1.2 & 1.63 \\
\hline
\end{tabular}

Table 2: Errors for the mass determination in SPS1a, taken from $[6,7,19]$. Shown are the nominal parameter values and the experimental and sum of experimental and theoretical uncertainties for the LHC alone, the LC alone, and a combined LHC+LC analysis. All values are given in GeV. 


\begin{tabular}{|l|rr|rr|rr|rr|}
\hline & SPS1a & StartFit & LHC & $\Delta_{\text {LHC }}$ & LC & $\Delta_{\text {LC }}$ & LHC+LC & $\Delta_{\text {LHC }+ \text { LC }}$ \\
\hline$M_{0}$ & 100 & 500 & 100.0 & 3.9 & 100.0 & 0.08 & 100.04 & 0.08 \\
$M_{1 / 2}$ & 250 & 500 & 250.0 & 1.8 & 250.0 & 0.13 & 250.01 & 0.11 \\
$\tan \beta$ & 10 & 50 & 10.0 & 1.3 & 10.0 & 0.14 & 9.98 & 0.14 \\
$A_{0}$ & -100 & 0 & -100.0 & 38.3 & -100.0 & 4.40 & -98.25 & 4.13 \\
\hline
\end{tabular}

Table 3: Summary of the mSUGRA fits in SPS1a: true values, starting values, fit values and absolute errors from the fit. As in SPS1a we fix $\mu>0$. The mass values of the fits are based on values found in Tab. 2 taken with experimental uncertainties.

\begin{tabular}{|l|rr|rr|rr|rr|}
\hline & SPS1a & StartFit & LHC & $\Delta_{\text {LHC }}$ & LC & $\Delta_{\text {LC }}$ & LHC+LC & $\Delta_{\text {LHC }+ \text { LC }}$ \\
\hline$M_{0}$ & 100 & 500 & 100.0 & 4.6 & 100.0 & 0.70 & 100.0 & 0.68 \\
$M_{1 / 2}$ & 250 & 500 & 250.0 & 2.8 & 250.0 & 0.72 & 250.0 & 0.67 \\
$\tan \beta$ & 10 & 50 & 10.0 & 3.4 & 10.0 & 0.49 & 10.0 & 0.49 \\
$A_{0}$ & -100 & 0 & -100.0 & 50.5 & -100.0 & 13.9 & -100.0 & 13.1 \\
\hline
\end{tabular}

Table 4: Summary of the mSUGRA fits in SPS1a: true values, starting values, fit values and absolute errors from the fit. As in SPS1a we fix $\mu>0$. The mass values of the fits are based on values found in Tab. 2 taken with experimental and theoretical uncertainties.

\section{2 mSUGRA/cMSSM Parameter Determination}

Assuming that SUSY breaking is mediated by gravitational interactions (mSUGRA/cMSSM) we fit four universal high-scale parameters to a toy set of collider measurements: the universal scalar and gaugino masses, $m_{0}, m_{1 / 2}$, the trilinear coupling $A_{0}$ and the ratio of the Higgs vacuum expectation values, $\tan \beta$. The sign of the Higgsino mass parameter $\mu$ is a discrete parameter and therefore fixed. In contrast to an earlier study [15] we assume the set of mass measurement at the LHC and at the LC, shown in Tab. 2. The central value for our assumed data set corresponds to the SUSY parameter point SPS1a [20], as computed by SUSPECT. As mentioned in the introduction correlations, systematic errors and theoretical errors are neglected. As the central (true) values are used as measurements in order to study the errors on the determination of the parameters, the $\chi^{2}$ values are not meaningful and therefore are not quoted.

The starting points for the mSUGRA parameters are fixed to the mean of the lower and upper limit (typically $1 \mathrm{TeV} / \mathrm{c}^{2}$ ) of the allowed parameter range, i.e. they are not necessarily close to the true SPS1a values. The result of the fit is shown in Tab. 3 and Tab. 4. All true parameter values are reconstructed well within the quoted errors, in spite of starting values relatively far away. The measurements of $m_{0}$ and $m_{1 / 2}$ are very precise, while the sensitivity of the masses on $\tan \beta$ and $A_{0}$ is significantly weaker. The results for the LHC alone are generally an order of magnitude less precise than those for the LC, and this qualitative difference is expected to become even more pronounced once we properly include systematical errors.

Because the data set is fit assuming mSUGRA as a unification scenario the absence of measurements of most of the strongly interacting particles, in particular the gluino, does not have a strong impact on the precision of the LC determination. Therefore the results for the combined measurements LHC+LC show only a small improvement.

Assuming an uncorrelated data set, the correlations between the different high-scale 


\begin{tabular}{|l|rrr|r|r|r|}
\hline & SPS1a & Start & Fit & $\Delta_{\text {LHC }}$ & $\Delta_{\text {LC }}$ & $\Delta_{\text {LHC+LC }}$ \\
\hline$M_{0}$ & 100 & 500 & 100.0 & 4.6 & 0.70 & 0.68 \\
$M_{1 / 2}$ & 250 & 500 & 250.0 & 2.8 & 0.72 & 0.67 \\
$\tan \beta$ & 10 & 50 & 10.0 & 3.4 & 0.49 & 0.49 \\
$A_{0}$ & -100 & 0 & -100.0 & 50.5 & 13.9 & 13.1 \\
\hline
\end{tabular}

Table 5: Summary of the mSUGRA fits in SPS1a: true values, starting values, fit values and absolute errors from the fit. As in SPS1a we fix $\mu>0$. The mass values of the fits are based on values found in Tab. 2 taken with experimental and theoretical uncertainties.

\begin{tabular}{|l|rr|rr|rr|}
\hline LHC & Suspect & $\Delta$ & Softsusy & $\Delta$ & IsaSusy & $\Delta$ \\
\hline$M_{0}$ & 100.0 & 4.6 & 97.9 & 4.1 & 99.7 & 4.4 \\
$M_{1 / 2}$ & 250.0 & 2.8 & 252.5 & 2.0 & 252.3 & \\
$\tan \beta$ & 10.0 & 3.4 & 11.6 & 3.4 & 9.2 & 2.4 \\
$A_{0}$ & -100.0 & 50.5 & 14.7 & 14.2 & -34.3 & 8.4 \\
\hline \hline LC & Suspect & $\Delta$ & Softsusy & $\Delta$ & IsaSusy & $\Delta$ \\
\hline$M_{0}$ & 100.0 & 0.7 & 98.7 & 0.7 & 100.6 & 0.5 \\
$M_{1 / 2}$ & 250.0 & 0.7 & 250.7 & 0.7 & 252.7 & \\
$\tan \beta$ & 10.0 & 0.5 & 10.1 & 0.5 & 9.95 & 0.4 \\
$A_{0}$ & -100.0 & 14 & -45.2 & 16 & -71.7 & 9.6 \\
\hline \hline LHC+LC & Suspect & $\Delta$ & Softsusy & $\Delta$ & IsaSusy & $\Delta$ \\
\hline$M_{0}$ & 100.0 & 0.7 & 98.5 & 0.8 & 100.7 & 0.6 \\
$M_{1 / 2}$ & 250.0 & 0.7 & 250.9 & 0.8 & 252.7 & \\
$\tan \beta$ & 10.0 & 0.5 & 10.1 & 0.6 & 9.9 & 0.3 \\
$A_{0}$ & -100.0 & 13 & -43.4 & 17 & -70.1 & 8.5 \\
\hline
\end{tabular}

Table 6:

SUSY parameters which we obtain from the fit are given in Tab. 8. We can understand the correlation matrix step by step [21]: first, the universal gaugino mass $m_{1 / 2}$ can be extracted very precisely from the physical gaugino masses. The determination of the universal scalar mass $m_{0}$ is dominated by the weak-scale scalar particle spectrum, but in particular the squark masses are also strongly dependent on the universal gaugino mass, because of mixing effects in the renormalization group running. Hence, a strong correlation between the $m_{0}$ and $m_{1 / 2}$ occurs. The universal trilinear coupling $A_{0}$ can be measured through the third generation weak-scale mass parameters $A_{b, t, \tau}$. However, the $A_{b, t, \tau}$ which appear for example in the off-diagonal elements of the scalar mass matrices, also depend on $m_{0}$ and $m_{1 / 2}$, so that $A_{0}$ is strongly correlated with $m_{0}$ and $m_{1 / 2}$. At this point one should stress that the determination of $A_{0}$ is likely to be dominated by $A_{t}$ as it appears in the calculation of the lightest Higgs mass $m_{h}$. After taking into account the current theoretical error of $3 \mathrm{GeV}$ on $m_{h}$ [22] we expect the determination of $A_{0}$ to suffer significantly. The experimental errors therefore can be considered a call for an improvement of the theoretical error.

In general, $\tan \beta$ can be determined in three sectors of the supersymmetric spectrum: all four Higgs masses, and for large values of $m_{A}$ in particular the light $\mathrm{CP}$ even Higgs mass $m_{h}$ depend on $\tan \beta$. The mixing between gauginos and Higgsinos in the neutralino/chargino sector is governed by $\tan \beta$. Finally, the stop mixing is governed by 


\begin{tabular}{|l|rr|rr|}
\hline LHC & Suspect & $\Delta$ & Softsusy & $\Delta$ \\
\hline$M_{0}$ & 100.0 & 4.6 & 97.9 & 4.1 \\
$M_{1 / 2}$ & 250.0 & 2.8 & 252.5 & 2.0 \\
$\tan \beta$ & 10.0 & 3.4 & 11.6 & 3.4 \\
$A_{0}$ & -100.0 & 50.5 & 14.7 & 14.2 \\
\hline \hline LC & Suspect & $\Delta$ & Softsusy & $\Delta$ \\
\hline$M_{0}$ & 100.0 & 0.7 & 98.7 & 0.7 \\
$M_{1 / 2}$ & 250.0 & 0.7 & 250.7 & 0.7 \\
$\tan \beta$ & 10.0 & 0.5 & 10.1 & 0.5 \\
$A_{0}$ & -100.0 & 14 & -45.2 & 16 \\
\hline \hline LHC+LC & Suspect & $\Delta$ & Softsusy & $\Delta$ \\
\hline$M_{0}$ & 100.0 & 0.7 & 98.5 & 0.8 \\
$M_{1 / 2}$ & 250.0 & 0.7 & 250.9 & 0.8 \\
$\tan \beta$ & 10.0 & 0.5 & 10.1 & 0.6 \\
$A_{0}$ & -100.0 & 13 & -43.4 & 17 \\
\hline
\end{tabular}

Table 7:

\begin{tabular}{|c|rrrr|}
\hline & $m_{0}$ & $m_{1 / 2}$ & $\tan \beta$ & $A_{0}$ \\
\hline$m_{0}$ & 1.000 & -0.602 & 0.422 & -0.424 \\
$m_{1 / 2}$ & & 1.000 & -0.250 & 0.636 \\
$\tan \beta$ & & & 1.000 & -0.241 \\
$A_{0}$ & & & & 1.000 \\
\hline
\end{tabular}

Table 8: The (symmetric) correlation matrix for the mSUGRA fit given in Tab.4 with data set $\mathrm{LHC}+\mathrm{LC}$.

$\mu / \tan \beta$, while the sbottom and stau mixing depends on $\mu \tan \beta$. The correlation of $\tan \beta$ with the other model parameters reflects the relative impact of these three sectors. In an earlier analysis we assumed a uniform error of $0.5 \%$ on all mass measurements [15] and saw that in this case $\tan \beta$ is determined through stau mixing, which in turn means that it shows very little correlation with $m_{1 / 2}$.

For the more realistic scenario in Tab. 2 the outcome is the following: the relative errors for the light Higgs mass and for the light neutralino masses at the LC are tiny. The relevant parameter in the Higgs sector is the light stop mass, which is governed by $m_{1 / 2}$; similarly the gaugino mass $m_{1 / 2}$ which fixes the light neutralino and chargino masses does not depend strongly on $\tan \beta$. The slepton sector introduces a strong correlation between $m_{0}$ and $m_{1 / 2}$. The resulting correlation matrix is shown in in Tab. 8. The results obtained with SFITTER are in agreement with expectation.

\section{General MSSM Parameter Determination}

In this study, the unconstrained weak-scale MSSM is described by 24 parameters in addition to the standard model parameters. The parameters are listed in Tab. 9: $\tan \beta$ as in mSUGRA, plus three soft SUSY breaking gaugino masses $M_{i}$, the Higgsino mass parameter $\mu$, the pseudoscalar Higgs mass $m_{A}$, the soft SUSY breaking masses for the right sfermions, $M_{\tilde{f}_{R^{\prime}}}$ the corresponding masses for the left doublet sfermions, $M_{\tilde{f}_{L}}$ and finally 


\begin{tabular}{|l|rrr||l|rrr|}
\hline & AfterGrid & AfterFit & SPS1a & & AfterGrid & AfterFit & SPS1a \\
\hline $\tan \beta$ & 100 & $10.02 \pm 3.4$ & 10 & $M_{\tilde{u}_{R}}$ & 532.1 & $532.1 \pm 2.8$ & 532.1 \\
$M_{1}$ & 100 & $102.2 \pm 0.74$ & 102.2 & $M_{\tilde{d}_{R}}$ & 529.3 & $529.3 \pm 2.8$ & 529.3 \\
$M_{2}$ & 200 & $191.79 \pm 1.9$ & 191.8 & $M_{\tilde{c}_{R}}$ & 532.1 & $532.1 \pm 2.8$ & 532.1 \\
$M_{3}$ & 589.4 & $589.4 \pm 7.0$ & 589.4 & $M_{\tilde{s}_{R}}$ & 529.3 & $529.3 \pm 2.8$ & 529.3 \\
$\mu$ & 300 & $344.3 \pm 1.3$ & 344.3 & $M_{\tilde{t}_{R}}$ & 420.2 & $420.08 \pm 13.3$ & 420.2 \\
$m_{A}$ & 399.35 & $399.1 \pm 1.2$ & 399.1 & $M_{\tilde{b}_{R}}$ & 525.6 & $525.5 \pm 10.1$ & 525.6 \\
$M_{\tilde{e}_{R}}$ & 138.2 & $138.2 \pm 0.76$ & 138.2 & $M_{\tilde{q} 1_{L}}$ & 553.7 & $553.7 \pm 2.1$ & 553.7 \\
$M_{\tilde{\mu}_{R}}$ & 138.2 & $138.2 \pm 0.76$ & 138.2 & $M_{\tilde{q} 2_{L}}$ & 553.7 & $553.7 \pm 2.1$ & 553.7 \\
$M_{\tilde{\tau}_{R}}$ & 135.5 & $135.48 \pm 2.3$ & 135.5 & $M_{\tilde{q} 3_{L}}$ & 501.3 & $501.42 \pm 10$. & 501.3 \\
$M_{\tilde{e}_{L}}$ & 198.7 & $198.7 \pm 0.68$ & 198.7 & $A_{\tau}$ & -253.5 & $-244.7 \pm 1428$ & -253.5 \\
$M_{\tilde{\mu}_{L}}$ & 198.7 & $198.7 \pm 0.68$ & 198.7 & $A_{t}$ & -504.9 & $-504.62 \pm 27$. & -504.9 \\
$M_{\tilde{\tau}_{L}}$ & 197.8 & $197.81 \pm 0.92$ & 197.8 & $A_{b}$ & -797.99 & $-825.2 \pm 2494$ & -799.4 \\
\hline
\end{tabular}

Table 9: Result for the general MSSM parameter determination in SPS1a using the toy sample of all MSSM particle masses with a universal error of $0.5 \%$. Shown are the nominal parameter values, the result after the grid and the final result. All masses are given in GeV.

the trilinear couplings of the third generation sfermions $A_{t, b, \tau}$.

\subsection{Toy model with all masses}

For testing purposes, we first consider a toy data set which includes all supersymmetric particle masses. The universal error on all mass measurements is set to $0.5 \%$.

In any MSSM spectrum, in first approximation, the parameters $M_{1}, M_{2}, \mu$ and $\tan \beta$ determine the neutralino and chargino masses and couplings. We exploit this feature to illustrate the option to use a grid before starting the fit. The starting values of the parameters other than $M_{1}, M_{2}, \mu$ and $\tan \beta$ are set to their nominal values, this study is thus less general than the one of mSUGRA. The $\chi^{2}$ is then minimized on a grid using the six chargino and neutralino masses as measurements to determine the four parameters $M_{1}$, $M_{2}, \mu$ and $\tan \beta$. The step size of the grid is 10 for $\tan \beta$ and $100 \mathrm{GeV}$ for the mass parameters. After the minimization, the four parameters obtained from grid minimization are fixed and all remaining parameters are fitted. In a final run all model parameters are released and fitted. The results after the grid (including the complementary fit), after the final fit and the nominal values are shown in Tab. 9. The smearing option has not been applied. However, the errors on the fitted values (once the fit converges) should not be sensitive to these shortcomings.

The final fit values indeed converges to the correct central values within its error. The central values of the fit are in good agreement with generated values, except for the trilinear coupling $A_{b, \tau}$. The problem is using only mass measurements to determine the three entries in a (symmetric) scalar mass matrix: in the light slepton sector there are three masses, left and right scalars plus the sneutrino, so the system is in principle calculable. In the third generation squark sector we have three independent diagonal entries per generation and two off-diagonal entries. But the number of mass measurements is only four, therefore the system is underdetermined in first order. The off-diagonal entry in the mass matrix for down type scalars includes a term $A_{b, \tau}$ and an additional term $\mu \tan \beta$. Even for very moderate values of $\tan \beta$ the extraction of $A_{b, \tau}$ requires precise knowledge 
of $\tan \beta$. The use of branching ratios and cross section measurements (with polarised beams) which carry information about the scalar mixing angles should significantly improve the determination of $A_{t, b, \tau}$.

\subsection{Toy model with LHC+LC mass measurements}

\begin{tabular}{|l|ccc|c|}
\hline & $\Delta_{L H C}$ & $\Delta_{L C}$ & $\Delta_{L H C+L C}$ & SPS1a value \\
\hline $\tan \beta$ & 9.1 & 0.3 & 0.2 & 10 \\
$M_{1}$ & 5.3 & 0.1 & 0.1 & 102.2 \\
$M_{2}$ & 7.3 & 0.7 & 0.2 & 191.8 \\
$M_{3}$ & 15 & fixed 500 & 11 & 589.4 \\
$M_{\tilde{\tau}_{L}}$ & fixed 500 & 1.2 & 1.1 & 197.8 \\
$M_{\tilde{\tau}_{R}}$ & 6.9 & 0.3 & 0.6 & 135.5 \\
$M_{\tilde{\mu}_{L}}$ & 5.1 & 0.5 & 0.5 & 198.7 \\
$M_{\tilde{\mu}_{R}}$ & 5.0 & 0.2 & 0.2 & 138.2 \\
$M_{\tilde{e}_{L}}$ & 5.1 & 0.2 & 0.2 & 198.7 \\
$M_{\tilde{e}_{R}}$ & 5.0 & 0.05 & 0.05 & 138.2 \\
$M_{\tilde{q} 3_{L}}$ & 110 & 4.4 & 39 & 501.3 \\
$M_{\tilde{t}_{R}}$ & fixed 500 & 2.1 & 12 & 420.2 \\
$M_{\tilde{b}_{R}}$ & 113 & fixed 500 & 61 & 525.6 \\
$M_{\tilde{q} 2_{L}}$ & 13 & fixed 500 & 5.5 & 553.7 \\
$M_{\tilde{c}_{R}}$ & 20 & fixed 500 & 15 & 532.1 \\
$M_{\tilde{s}_{R}}$ & 20 & fixed 500 & 15 & 529.3 \\
$M_{\tilde{q} 1_{L}}$ & 13 & fixed 500 & 6.5 & 553.7 \\
$M_{\tilde{u}_{R}}$ & 20 & fixed 500 & 15 & 532.1 \\
$M_{\tilde{d}_{R}}$ & 20 & fixed 500 & 15 & 529.3 \\
$A_{\tau}$ & fixed 0 & 89.5 & 171 & -253.5 \\
$A_{t}$ & 91 & 2.7 & 3.3 & -504.9 \\
$A_{b}$ & 35600 & fixed 0 & 12500 & -799.4 \\
$m_{A}$ & fixed 500 & 0.9 & 0.8 & 399.1 \\
$\mu$ & 7.3 & 2.3 & 1.0 & 344.3 \\
\hline
\end{tabular}

Table 10: Result for the general MSSM parameter determination in SPS1a using the mass measurements given in Tab. 2 with experimental errors only. Shown are the nominal parameter values and the result after fits to the different data sets. All masses are given in $\mathrm{GeV}$.

In the study of the three data sets LHC, LC, and LHC+LC in the MSSM, a fit was performed for the data sets LHC and LC, whereas for LHC+LC additionally the GRID was used for $M_{1}, M_{2}, \mu$ and $\tan \beta$ with the five chargino and neutralino masses. The starting points were chosen to be the true values (with the exception of the parameters used in the grid). In order to obtain a solvable system, for the LHC data set $m_{A}, M_{\tilde{t}_{R^{\prime}}}$ $M_{\tilde{\tau}_{L}}, A_{\tau}$ were fixed. For the LC data set the first and second generation squark soft SUSY breaking masses, the gluino mass $M_{3}, M_{\tilde{b}_{R}}$ and $A_{b}$ were fixed. These parameters were chosen on the basis of the measurements available in Tab. 2. The values to which these parameters were fixed is not expected to influence the final result of the fit. The results for the two data sets are shown in Tab. 10 and Tab. 13. 


\begin{tabular}{|l|ccc|c|}
\hline & $\Delta_{L H C}$ & $\Delta_{L C}$ & $\Delta_{L H C+L C}$ & SPS1a value \\
\hline $\tan \beta$ & 11 & 6.1 & 5.5 & 10 \\
$M_{1}$ & 5.3 & 1.2 & 0.9 & 102.2 \\
$M_{2}$ & 8.2 & 3.6 & 3.2 & 191.8 \\
$M_{3}$ & 20 & fixed 500 & 21 & 589.4 \\
$M_{\tilde{\tau}_{L}}$ & fixed 500 & 8.9 & 8.7 & 197.8 \\
$M_{\tilde{\tau}_{R}}$ & 16 & 13 & 12 & 135.5 \\
$M_{\tilde{\mu}_{L}}$ & 5.1 & 1.3 & 1.25 & 198.7 \\
$M_{\tilde{\mu}_{R}}$ & 5.0 & 0.7 & 0.7 & 138.2 \\
$M_{\tilde{e}_{L}}$ & 5.1 & 0.9 & 0.9 & 198.7 \\
$M_{\tilde{e}_{R}}$ & 5.0 & 0.7 & 0.65 & 138.2 \\
$M_{\tilde{q} 3_{L}}$ & 110 & 330 & 21 & 501.3 \\
$M_{\tilde{t}_{R}}$ & fixed 500 & 160 & 83 & 420.2 \\
$M_{\tilde{b}_{R}}$ & 112 & fixed 500 & 21 & 525.6 \\
$M_{\tilde{q} 2_{L}}$ & 13 & fixed 500 & 17 & 553.7 \\
$M_{\tilde{c}_{R}}$ & 20 & fixed 500 & 23 & 532.1 \\
$M_{\tilde{s}_{R}}$ & 20 & fixed 500 & 23 & 529.3 \\
$M_{\tilde{q} 1_{L}}$ & 13 & fixed 500 & 16 & 553.7 \\
$M_{\tilde{u}_{R}}$ & 20 & fixed 500 & 23 & 532.1 \\
$M_{\tilde{d}_{R}}$ & 20 & fixed 500 & 23 & 529.3 \\
$A_{\tau}$ & fixed 0 & 2900 & 3160 & -253.5 \\
$A_{t}$ & 106 & 140 & 126 & -504.9 \\
$A_{b}$ & 35100 & fixed 0 & fixed 0 & -799.4 \\
$m_{A}$ & fixed 500 & 2.0 & 2.0 & 399.1 \\
$\mu$ & 8.2 & 3.2 & 3.0 & 344.3 \\
\hline
\end{tabular}

Table 11: Result for the general MSSM parameter determination in SPS1a using the mass measurements given in Tab. 2 with experimental and theoretical errors. Shown are the nominal parameter values and the result after fits to the different data sets. All masses are given in $\mathrm{GeV}$.

Note that the general rule that the LHC is not sensitive to weakly interacting particle masses is not entirely true: while the LHC has the advantage of measuring the squark and gluino masses, the first and second generation slepton mass parameters are also determined with a precision of the order of percent. The results in Tab. 13 show that the LHC alone is well capable of determining for example all gaugino mass parameters as well as most of the scalar mass parameters.

The situation at the LC is slightly different. Only marginal information on the squark sector available at the LC. The measurement of $A_{t}$ from the Higgs sector should be taken with a grain of salt (theoretical error on the lightest Higgs mass). Adding the stau mixing angle to the set of LC measurements will improve the determination of $A_{\tau}$. However, the measurements of the parameters, in particular slepton and gaugino parameters are far more precise than at the LHC.

For the LHC $+\mathrm{LC}$ data set, a sufficient number of mass measurements is available, so that no parameters need to be fixed. The superiority of the combination of the measurements at the two colliders is obvious from this observation and from Tab. 13: The LHC contributes to reduce the error in the weak sector $\left(M_{2}\right)$ and the LC in the strongly interacting sector (third generation squarks). Even more important: of 13 parameters un- 


\begin{tabular}{|l|ccc|c|}
\hline & $\Delta_{L H C}$ & $\Delta_{L C}$ & $\Delta_{L H C+L C}$ & SPS1a value \\
\hline $\tan \beta$ & 11 & 6.1 & 5.5 & 10 \\
$M_{1}$ & 5.3 & 1.2 & 0.9 & 102.2 \\
$M_{2}$ & 8.2 & 3.6 & 3.2 & 191.8 \\
$M_{3}$ & 20 & fixed 500 & 21 & 589.4 \\
\hline$m_{A}$ & fixed 500 & 2.0 & 2.0 & 399.1 \\
$\mu$ & 8.2 & 3.2 & 3.0 & 344.3 \\
\hline
\end{tabular}

Table 12: Result for the general MSSM parameter determination in SPS1a using the mass measurements given in Tab. 2 with experimental and theoretical errors. Shown are the nominal parameter values and the result after fits to the different data sets. All masses are given in $\mathrm{GeV}$.

determined by either the LHC or the LC, 11 are determined with good precision in the combination. For $A_{\tau}, A_{b}$, we expect an improvement with the use of branching ratios and cross section measurements.

A complete measurement of all parameters at the weak scale is particularly important if one wants to probe unification scenarios which link subsectors of the parameter space which are independent at the weak scale. An advanced tool like SFITTER can extract the information to probe supersymmetry breaking scenarios from any set of measurements, provided the set is sufficient to overconstrain the model parameters.

\section{Conclusions}

SFITTER is a new program to determine supersymmetric parameters from measurements. The parameters can be extracted either using a fit, a multi-dimensional grid, or a combination of the two. Correlations between measurements can be specified and are taken into account. While it is relatively easy to fit a fixed model with very few parameters for example at a high scale to a set of collider measurements, the determination of the complete set of weak-scale MSSM model parameters requires this more advanced tool. A mSUGRA inspired fit does not include the full complexity and power of the combined LHC and LC data compared to the measurements at either collider alone. The results from SFITTER in the MSSM with the three data sets show that only the combination of measurements of both the LHC and the LC offers a complete picture of the MSSM model parameters in a reasonably model independent framework.

\section{Acknowledgements}

The authors would like to thank the organizers of the Les Houches workshop and the convenors of the SUSY-group for the constructive atmosphere in which SFITTER was born.

\section{References}

[1] J. Wess and B. Zumino, Nucl. Phys. B 70 (1974) 39. 


\begin{tabular}{|l|ccc|c|}
\hline & $\Delta_{L H C}$ & $\Delta_{L C}$ & $\Delta_{L H C+L C}$ & SPS1a value \\
\hline$M_{\tilde{\tau}_{L}}$ & fixed 500 & 8.9 & 8.7 & 197.8 \\
$M_{\tilde{\tau}_{R}}$ & 16 & 13 & 12 & 135.5 \\
$M_{\tilde{\mu}_{L}}$ & 5.1 & 1.3 & 1.25 & 198.7 \\
$M_{\tilde{\mu}_{R}}$ & 5.0 & 0.7 & 0.7 & 138.2 \\
$M_{\tilde{e}_{L}}$ & 5.1 & 0.9 & 0.9 & 198.7 \\
$M_{\tilde{e}_{R}}$ & 5.0 & 0.7 & 0.65 & 138.2 \\
\hline$M_{\tilde{q} 3_{L}}$ & 110 & 330 & 21 & 501.3 \\
$M_{\tilde{t}_{R}}$ & fixed 500 & 160 & 83 & 420.2 \\
$M_{\tilde{b}_{R}}$ & 112 & fixed 500 & 21 & 525.6 \\
$M_{\tilde{q} 2_{L}}$ & 13 & fixed 500 & 17 & 553.7 \\
$M_{\tilde{c}_{R}}$ & 20 & fixed 500 & 23 & 532.1 \\
$M_{\tilde{s}_{R}}$ & 20 & fixed 500 & 23 & 529.3 \\
$M_{\tilde{q} 1_{L}}$ & 13 & fixed 500 & 16 & 553.7 \\
$M_{\tilde{u}_{R}}$ & 20 & fixed 500 & 23 & 532.1 \\
$M_{\tilde{d}_{R}}$ & 20 & fixed 500 & 23 & 529.3 \\
\hline$A_{\tau}$ & fixed 0 & 2900 & 3160 & -253.5 \\
$A_{t}$ & 106 & 140 & 126 & -504.9 \\
$A_{b}$ & 35100 & fixed 0 & 15600 & -799.4 \\
\hline
\end{tabular}

Table 13: Result for the general MSSM parameter determination in SPS1a using the mass measurements given in Tab. 2 with experimental and theoretical errors. Shown are the nominal parameter values and the result after fits to the different data sets. All masses are given in $\mathrm{GeV}$.

[2] G. A. Blair, W. Porod and P. M. Zerwas, Eur. Phys. J. C 27 (2003) 263 [arXiv:hepph/0210058].

[3] ATLAS Collaboration, Detector and Physics Performance TDR, Vol. II, CERN/LHC/99-15; TESLA Technical Design Report (Part 3), R.D. Heuer, D.J. Miller, F. Richard and P.M. Zerwas (eds.), DESY 010-11 [arXiv:hep-ph/0106315]; American LC Working Group, T. Abe et al., SLAC-R-570 (2001), [arXiv:hep-ex/0106055-58]; ACFA LC Working Group, K. Abe et al., KEK-REPORT-2001-11, [arXiv:hepex/0109166].

[4] S. Y. Choi, A. Djouadi, M. Guchait, J. Kalinowski, H.S. Song and P. M. Zerwas, Eur. Phys. J. C 14 (2000) 535 [arXiv:hep-ph/0002033]; V. D. Barger, T. Han, T. J. Li and T. Plehn, Phys. Lett. B 475 (2000) 342 [arXiv:hep-ph/9907425]; S. Y. Choi, J. Kalinowski, G. Moortgat-Pick and P. M. Zerwas, Eur. Phys. J. C 22 (2001) 563 [Addendum-ibid. C 23 (2002) 769] [arXiv:hep-ph/0108117]; K. Desch, J. Kalinowski, G. Moortgat-Pick, M. M. Nojiri and G. Polesello, arXiv:hep-ph/0312069.

[5] P. Fayet and J. Iliopoulos, Phys. Lett. B 51 (1974) 461.

[6] B. C. Allanach and others, hep-ph/0407067

[7] B. C. Allanach, A. Djouadi, J. L. Kneur, W. Porod and P. Slavich, hep-ph/0406166

[8] A. Djouadi, J. L. Kneur and G. Moultaka, arXiv:hep-ph/0211331. 
[9] P. Skands et al., arXiv:hep-ph/0311123.

[10] G. Ganis, MSMlib (unpublished)

[11] V. D. Barger, T. Falk, T. Han, J. Jiang, T. Li and T. Plehn, Phys. Rev. D 64 (2001) 056007 [arXiv:hep-ph/0101106].

[12] W. Beenakker, R. Hopker, M. Spira and P. M. Zerwas, Nucl. Phys. B 492 (1997) 51 [arXiv:hep-ph/9610490]; W. Beenakker, M. Kramer, T. Plehn, M. Spira and P. M. Zerwas, Nucl. Phys. B 515 (1998) 3 [arXiv:hep-ph/9710451]; W. Beenakker, M. Klasen, M. Kramer, T. Plehn, M. Spira and P. M. Zerwas, Phys. Rev. Lett. 83 (1999) 3780 [arXiv:hep-ph/9906298].

[13] F. James and M. Roos, Comput. Phys. Commun. 10 (1975) 343.

[14] M. Muhlleitner, A. Djouadi and Y. Mambrini, arXiv:hep-ph/0311167.

[15] R. Lafaye, T. Plehn and D. Zerwas, Prepared for Workshop on Physics at TeV Colliders, Les Houches, France, 2003, LAPP-EXP-2003-27, CERN-TH/2003-312, LAL 04/06

[16] B. C. Allanach, Comput. Phys. Commun. 143 (2002) 305 [arXiv:hep-ph/0104145].

[17] H. Baer, F. E. Paige, S. D. Protopescu and X. Tata, arXiv:hep-ph/0312045.

[18] W. Porod, Comput. Phys. Commun. 153 (2003) 275 [arXiv:hep-ph/0301101].

[19] B. Gjelsten et al., H.-U. Martyn, M. Chiorboli et al., all in this report.

[20] B. C. Allanach et al., in Proc. of the APS/DPF/DPB Summer Study on the Future of Particle Physics (Snowmass 2001) ed. N. Graf, Eur. Phys. J. C 25 (2002) 113 [eConf C010630 (2001) P125] [arXiv:hep-ph/0202233]; N. Ghodbane and H. U. Martyn, in Proc. of the APS/DPF/DPB Summer Study on the Future of Particle Physics (Snowmass 2001) ed. N. Graf, arXiv:hep-ph/0201233.

[21] M. Drees and S. P. Martin, arXiv:hep-ph/9504324.

[22] G. Degrassi, S. Heinemeyer, W. Hollik, P. Slavich and G. Weiglein, Eur. Phys. J. C 28 (2003) 133 [arXiv:hep-ph/0212020]. 